\title{
HABILIDADES DE LINGUAGEM E PESSOAL-SOCIAL DE CRIANÇAS DE 0 A 3 ANOS DE IDADE CUIDADAS EM CRECHES
}

\section{LANGUAGE AND PERSONAL-SOCIAL ABILITIES OF CHILDREN FROM 0 TO 3 YEARS OLD ASSISTED IN CHILD DAY CARE CENTERS}

Magda A. Rezende ${ }^{1,2}$

Fernanda G. de Lima $^{3}$

Vivian C. Beteli ${ }^{3}$

Jair Lício F. Santos ${ }^{4}$

REZENDE, M.A .; LIMA, F.G.d.; BETELI, V.C.; SANTOS, J.L.F. Habilidades de linguagem e pessoalsocial de crianças de 0 a 3 anos de idade cuidadas em creches. Rev. Bras. Cresc. Desenv. Hum., São Paulo, 2003.

Resumo: Supervisão e promoção do desenvolvimento infantil são ações de saúde importantes que devem ser realizadas em creches e pré-escolas. Estas ações podem propiciar o desenvolvimento da criança, especialmente em áreas em que é mais dependente do adulto, tais como as de linguagem e pessoal-social. Nosso objetivo no presente estudo foi avaliar habilidades de linguagem e pessoal-social de crianças que freqüentavam 3 creches da cidade de São Paulo. Trinta e três crianças, de 0 a 36 meses, 18 meninos e 15 meninas, foram avaliadas pelo Teste de Triagem de Desenvolvimento de Denver II em 2 momentos, separados entre si por 5 meses. As crianças eram de nível sócio-econômico homogêneo. Os fatores estruturais (proporção adulto/criança, grupos pequenos de crianças) das 3 creches eram adequados. O peso de nascimento variou de 1970 a $4280 \mathrm{~g}$. As classificações obtidas foram unificadas após análise não significante (teste de Fischer). Na segunda avaliação, as habilidades das crianças melhoraram nas duas áreas. Os resultados de cada criança foram emparelhados e analisados com o Teste dos Sinais (risco a $=0,05$ ): o resultado foi não significante na linguagem e significante na pessoal-social $(\mathrm{p}=0,008)$.Conclui-se que a creche deve ter contribuído para a obtenção destes resultados.

Palavras-chave: creches; educação infantil; cuidado da criança; enfermagem pediátrica; desen volvimento infantil; teste de triagem de desenvolvimento de Denver II.

\section{DESENVOLVIMENTO INFANTIL EM CRECHES}

Em instituições de cuidado profissional, como é o caso de creches - instituições de edu- cação infantil em que se atende crianças de 0 a 3 anos de idade -, impõe-se a necessidade de se promover condições para que o desenvolvimento infantil ocorra em sua plenitude, bem como avaliá-lo periodicamente, uma vez que

1 Pesquisa parcialmente financiada por Bolsa de Iniciação Científica fornecida pela FAPESP à Vivian C. Beteli (Processo 00/11890-7).

2 Professor Doutor do Departamento de enfermagem Materno-Infantil e Psiquiátrica da Escola de enfermagem da USP. Coordenadora do grupo de pesquisas “Saúde em creches e saúde da criança” (Diretório de Grupos de Pesquisas do CNPq - versão 5.0).

3 Alunas do curso de graduação em enfermagem da Escola de Enfermagem da USP (EEUSP).

4 Professor Titular do Departamento de Medicina Social da Faculdade de Medicina da USP de Ribeirão Preto. 
isto permite uma rápida tomada de decisões, caso necessário (WONG, 1999). A “supervisão do desenvolvimento" é tão importante que tem sido periodicamente sistematizada por organismos internacionais de saúde, como a OPAS (ORGANIZACIÓN PANAMERICANA DE LA SALUD, 1999), além do próprio Ministério da Saúde (MINISTÉRIO DA SAÚDE, 1995; MINISTÉRIO DA SAÚDE, 2002). Estas tentativas têm sido organizadas para serem utilizadas quando a criança é levada às consultas de saúde. No entanto, ainda não foi sistematizada uma estratégia para ser usada especificamente em instituições de educação infantil como creches e pré-escolas. Apesar disso, profissionais de enfermagem, bem como de outras áreas da saúde, já vêm se debruçando sobre o tema supervisão do desenvolvimento infantil em creches (BRÊTAS, 1991; BRÊTAS; SILVA, 1995; CUNHA, 2000; FERREIRA, 1989; HUGHES, 1986; KAKEHASHI, 1990; FISBERG et al., 1997).

A situação das crianças cuidadas em creche é especialmente importante, uma vez que nela permanecem grande parte de sua infância. Exemplo disto é a estimativa do Conselho Nacional de Credenciamento de Creches da Austrália (AUSTRÁLIA, 1993): uma criança de 5 anos pode freqüentar a creche durante 12.500 horas em um período de 5 anos (50 semanas $\mathrm{x}$ 50 horas) enquanto, para a sua escolarização, terá dispensado praticamente o mesmo número de horas: 13.000 (40 semanas x 25 horas $\mathrm{x}$ 13 anos).

No caso do Brasil, este contraste é ainda mais evidente pois a escolarização de $1^{\circ}$ e de $2^{\circ}$ graus soma 11 anos. Além disto, nossas crianças permanecem na instituição 8 horas por dia ou mais. Em 2001, 5.912.150 crianças freqüentavam 92.526 creches e pré-escolas5. É um grande contingente e ainda está aumentando.

\section{CRIANÇA, DESENVOLVIMENTO E CRECHE}

A escolha da creche, nesta investigação, deve-se ao fato de ser esta um local de cuidado humano profissional. Consideramos crucial analisar as influências ambientais colocadas à disposição de nossas crianças, deixando claro que entendemos como ambiente não somente os componentes físicos/arquitetônicos, mas também as pessoas que cuidam e educam. Percebe-se, portanto, a importância de se organizar as situações de cuidado, de molde a propiciar à criança oportunidades para se conhecer e se construir ativamente.

A criança deve encontrar no ambiente os componentes adequados a fim de que se desenvolva. Isto implica em dar-lhe condições quer de aceitação de sua singularidade, quer de apoio e de "desafio" para a aquisição de habilidades e conhecimentos segundo o estágio de sua maturação. A criança tem valor por si só e não apenas por sua "utilidade" (ou não) como profissional no futuro (VERÍSSIMO \& SIGAUD; 1996; TURNER, 2000).

A nossa experiência profissional mostrounos que a oportunidade de se desenvolver não vem sendo propiciada em creches que atendem os estratos econômicos mais baixos da população. Em uma situação de triagem, verificamos que o atraso de linguagem6 de crianças atendidas em creches públicas municipais de São Paulo, visto pelo Teste de Denver (WHALEY \& WONG, 1989) e pelo Guia Washington (BARNARD \& ERIKSON, 1978), era revertido em praticamente $100 \%$ destas crianças em duas semanas quando era propiciado um aumento de sua habilidade oral. Esta evidência foi repetida à exaustão. Contudo, não se trata de fenômeno restrito às crianças deste nível sócio-econômico. TOLEDO (2000) relata um trabalho realiza-

5 www.inep.gov.br/estatisticas/perfil/resp_uf_reg.asp?tipo=2\&regiao=BRASIL em 15-10-02.

6 O mesmo vêm sendo encontrado pela enfermeira e colega Damaris G. Maranhão ao avaliar desenvolvimento infantil em creches públicas da cidade de São Paulo (comunicação pessoal). 
do em escola de educação infantil privada da cidade de São Paulo. A estratégia de estimulação do uso da linguagem oral por crianças de 5 anos ocorreu por ter sido percebido que até mesmo crianças e adolescentes de 10 a 15 anos não sabiam comunicar-se sozinhos. Face a isto, esta autora propôs atividades para que as crianças daquela classe de pré-escola aumentassem suas habilidades. CARVALHO e SCARPA (2000) reforçam o tema afirmando que "embora a linguagem oral seja uma realidade presente na prática das instituições de educação infantil, ela nem sempre é tratada com a devida importância (......) [devido] à falta de conhecimento das reais competências da criança”.

Estamos propondo, portanto, o favorecimento do desenvolvimento como parte do cuidado de saúde à criança.

\section{OBJETIVO E METODOLOGIA}

O objetivo desta pesquisa foi avaliar as habilidades das áreas de linguagem e pessoalsocial de crianças de 0 a 3 anos de idade, que freqüentam creches consideradas de bom padrão de qualidade, em dois momentos separados por 5 meses de intervalo. Para tal foi utilizado o Teste de Triagem de Desenvolvimento de Denver II (ARCHER et al., 1992). Esperava-se que as crianças apresentassem um bom nível de desempenho já por ocasião da primeira avaliação, tendo em vista que as creches selecionadas para a pesquisa são patronais, isto é, destinadas ao acolhimento de filhos de funcionários da instituição donde pode-se supor uma boa escolaridade, condição relacionada a índices melhores de desenvolvimento (DURMAZLAR et al., 1998).

A segunda medição permitiu avaliar se as crianças haviam se mantido estáveis, melhorado ou piorado. Esperava-se que as crianças se mantivessem estáveis ou melhorado, uma vez que as creches foram consideradas adequadas.
Trata-se de um levantamento descritivo no qual são avaliadas crianças em dois momentos distintos e os dados comparados. Cada criança é seu próprio controle, ou seja, os dados são emparelhados.

\section{Cenário do estudo}

O estudo foi realizado em três creches consideradas de bom padrão de atendimento, localizadas próximas entre si, na cidade de São Paulo (creches A, B e C). No que diz respeito à qualidade de atendimento levou-se em conta a situação estrutural (tamanho das classes e proporção adulto/criança) uma vez que estas creches ainda estão sendo analisadas quanto ao processo (capacidade do educador de responder aos interesses e necessidades infantis, prover atividades adequadas ao desenvolvimento da criança e ter sensibilidade às suas necessidades, segundo BURCHINAL et al., 1996; BURCHINAL et al., 2000).

A escolha de creches consideradas adequadas permitirá, na indicação de alternativas no futuro, que se possa pontuar a necessidade de investimento em recursos humanos e materiaispara um cuidado/educação de boa qualidade.

\section{Grupo amostral}

Foi formado por crianças de 0 a 36 meses que preenchessem os requisitos gerais de: ter idade gestacional conhecida; não ter malformações congênitas; e não ser estrangeiras (devido à avaliação da linguagem).

As crianças eram de nível sócio-econômico homogêneo, segundo avaliação por formulário padronizado desenvolvido no Chile para verificação do nível de pobreza de populações urbanas (ALVAREZ et al., 1982) e adaptado para a realidade brasileira por ISSLER e GIUGLIANI (1997). O formulário consta de 13 itens, compreendendo: constituição da família, escolaridade e atividade dos pais, condições de 
moradia e posse de bens. Cada um dos itens estabelece uma pontuação, cuja soma estabelece o nível de pobreza: miséria (até 17,3 pontos), baixa inferior (17,3 a 34,6 pontos) e baixa superior (34,6 a 52,0 pontos).

As crianças foram avaliadas em dois momentos distintos, respeitando-se um intervalo mínimo de cinco meses entre as duas avaliações. Assim procedendo, esperava-se conseguir um período de tempo em que a criança pudesse adaptar-se à creche e ser ou não beneficiada por freqüentá-la.

\section{Método de avaliação: o Teste de Triagem de Desenvolvimento de Denver - II}

Para a avaliação das crianças foi usado o Teste de Denver, criado com a finalidade de triar de problemas de desenvolvimento. Elaborado em 1967, passou por uma reavaliação devido a algumas dificuldades na aplicação e interpretação. A partir disso, foi criado o TTDD-II (ARCHER et al., 1992) que pode ser aplicado em crianças desde o nascimento até 6 anos de idade. É composto por 125 itens, distribuídos em quatro áreas do desenvolvimento infantil: pessoal-social, motor fino-adaptativo, linguagem e motor-grosseiro. Alguns itens são aplicados através da solicitação para que a criança realize determinadas tarefas e outros através de relato de pais ou cuidadores/educadores da criança.

O TTDD-II, vem sendo considerado adequado para a identificação de até mesmo discretos problemas de desenvolvimento. Em nosso país, sabe-se que vem sendo usado por vários profissionais da área de saúde. Considerando que é instrumento bastante conhecido e usado em nossa área, optou-se por ele neste trabalho.

\section{Cuidados metodológicos na aplicação do TTDD-II}

Foram excluídas do grupo de pesquisa as crianças em que havia: (1) impossibilidade de conhecer a idade gestacional da criança (para as menores de 2 anos de idade), (2) malformação congênita, e, (3) ser criança estrangeira.

Outros fatores que também podiam levar à exclusão foram considerados: (1) os pais estarem se separando, (2) ter havido morte de familiar próximo, (3) ter ocorrido nascimento de irmão, (4) qualquer outro que fosse lembrada pela família ou pelos profissionais da creche, e que fosse por nós caracterizada como uma situação potencialmente estressante para a criança e, por conseguinte, para a avaliação de seu desenvolvimento.

Além disso, verificava-se as condições imediatamente anteriores à aplicação do teste. Estes eram fatores de exclusão de caráter transitório. Procurava-se saber se a criança estava: (1) com sono, (2) fatigada, (3) adoentada, (4) com febre, (5) ou com medo. Caso a criança apresentasse algum deles, esperava-se até que fosse superado. Isto podia demorar vários dias. Um formulário foi criado para este fim, a ser preenchido também com os dados: idade gestacional da criança (com respectiva necessidade de ajuste), cálculo de idade da criança, e peso ao nascer.

Durante a aplicação, caso a criança se cansasse, o teste era interrompido. Outra situação em que o teste também era interrompido acontecia quando a criança precisava participar de alguma atividade prevista na creche, por exemplo, jantar. Não seria possível ignorar estes momentos, pois a creche não teria condições de dar à criança um atendimento tão individualizado que levasse em conta, inclusive, mudança de horário de refeições. A aplicação do TTDD-II pode ser eventualmente muito demorada, especialmente neste cenário de estudo no qual, muitas vezes, mães, pais, ou outros cuidadores primários não estavam presentes. Assim, é crucial que estes cuidados sejam tomados a fim de que a criança esteja se sentindo absolutamente segura e que o desempenho avaliado seja o real. 


\section{Modo de aplicação do TTDD-II}

Antes da aplicação do teste é necessário realizar o cálculo da idade da criança em anos, meses e dias e traçar uma linha vertical correspondente a essa idade. Foi utilizado o formulário de aplicação traduzido e formatado por PEDROMÔNICO, BRAGATTO, STROBILIUS (1999). Aplicou-se todos os itens cortados pela linha e também três itens totalmente à esquerda em cada área do desenvolvimento, com o objetivo de detectar se havia atraso.

No cálculo da idade de crianças de até dois anos havia necessidade de se conhecer a idade gestacional. Caso a criança tivesse nascido prematuramente (menor ou igual a 37 semanas de gestação), a idade era ajustada de acordo com o recomendado pelos autores (ARCHER et al., 1992).

Para a aplicação do teste, são necessários os seguintes materiais: 1 pompom vermelho de 10 centímetros de diâmetro; 1 chocalho de cabo estreito; 1 sino; 1 saco de balas de goma, tipo "jujuba"; 10 cubos de madeira de 2,5 cm nas cores vermelho, amarelo, azul, verde e laranja; 1 caneca; 1 boneca de plástico com mamadeira; 1 lápis vermelho; folha de sulfite A4; 1 folha contendo cinco desenhos (pássaro, cachorro, gato, cavalo e menino) e folha de aplicação do teste.

Cada item aplicado pode ser classificado como: passou, falhou, não observado e recusa. Para a interpretação dos itens são utilizados os termos:

Normal: quando a criança passa em um item que está sendo cortado pela linha da idade, quando a criança falha ou recusa-se a realizar um item que está completamente à direita da linha da idade.

Cautela: quando a criança falha ou recusa-se a realizar o item no qual a linha da idade cruza entre 75 e $90 \%$.

Atraso: quando a criança falha ou recusa-se a realizar um item que esta totalmente a esquerda da linha da idade.
Nesta pesquisa o teste foi aplicado por inteiro, mas não foi interpretado como um todo, e sim em áreas isoladas. Cada área foi considerada adequada quando a criança não apresentou itens de cautela e/ou atraso.

Nas situações em que o resultado era suspeito (considerando o teste como um todo, ou seja, quando havia duas ou mais cautelas e/ ou um ou mais atrasos) as crianças eram reavaliadas uma ou duas semanas após.

\section{Análise dos dados e cuidados éticos}

A análise dos dados foi realizada através de testes não-paramétricos (SIEGEL, 1975).

A fim de que os participantes desta pesquisa tivessem seus direitos resguardados este projeto foi: (1) submetido à aprovação pela Comissão de Ética da Escola de Enfermagem da USP (EEUSP); (2) foi realizado esclarecimento às mães e pais (ou responsáveis) das crianças quanto ao objetivo da pesquisa, bem como a forma de realização. Foi garantido que as crianças individualmente não seriam identificadas nos relatórios produzidos. Após este esclarecimento, o responsável legal por cada criança, caso concordasse com a realização da pesquisa, assinava um Termo de Consentimento Livre e Esclarecido.

\section{APRESENTAÇÃO DOS RESULTADOS E DISCUSSÃO}

Na primeira avaliação foram analisadas 36 crianças. Destas, 19 são da Creche A, 9 da Creche B, e 8 da Creche C. Na segunda foram avaliadas 33, sendo 19 da creche A, 9 da creche B e 3 da creche $\mathrm{C}$. Vale lembrar que, para a primeira avaliação, muitas crianças não puderam ser consideradas por diferentes motivos, tais como, não autorização pela família (restrito a uma creche somente); criança sempre ausente nos períodos definidos para aplicação do teste; criança recusava-se a ser ava- 
liada; impossibilidade de contato com os pais a fim de completar os itens da avaliação; malformação congênita; desconhecimento da idade gestacional; criança estrangeira e criança que abandonava a creche após poucos dias de freqüência.

Na segunda avaliação, houve perda de três crianças, constituindo uma amostra final de 33. Os motivos foram: saída da creche e não comparecimento devido às férias da mãe.

Consideramos como idade da avaliação o momento em que esta de fato terminou de ser executada, uma vez que algumas foram realizadas em mais de um dia devido a peculiaridades da criança ou da creche. Além disso, havia a situação de, após uma primeira avaliação, a criança ser considerada suspeita em relação a alguma área. Neste caso, era feito uma retestagem cerca de uma ou duas semanas após. Deste modo evitou-se resultados falsamente negativos. Portanto, nas ocasiões de suspeita, a criança foi re-testada e esta é a data que estamos considerando. Houve necessidade de retestagem somente na primeira avaliação.

No momento que as crianças foram avaliadas estavam freqüentando a creche há períodos que variavam de 1 dia até 5 meses e 12 dias $\left(1^{\text {a }}\right.$ avaliação), e de 5 meses e 28 dias

Tabela 1. Resultados das Avaliações de Linguagem de Crianças das 3 Creches. São Paulo, 2001.

\begin{tabular}{cccccc}
\hline Avaliação & $\begin{array}{c}\text { Adequada } \\
\mathrm{n} \%\end{array}$ & $\begin{array}{c}\text { Cautela } \\
\mathrm{n} \%\end{array}$ & $\begin{array}{c}\text { Atraso } \\
\mathrm{n} \%\end{array}$ & $\begin{array}{c}\text { Cautela + Atraso } \\
\mathrm{n} \%\end{array}$ & $\begin{array}{c}\text { Total } \\
\mathrm{n} \%\end{array}$ \\
\hline $1^{\mathrm{a}}$ & 26 & 5 & 0 & 2 & 33 \\
& $(78,78)$ & $(15,15)$ & $(00,0)$ & $(6,07)$ & $(100,00)$ \\
\hline $2^{\circ}$ & 28 & 5 & 0 & 0 & 33 \\
& $(84,84)$ & $(15,15)$ & $(00,0)$ & $(00,0)$ & $(100,0)$ \\
\hline
\end{tabular}
até 9 meses e 13 dias ( $2^{\mathrm{a}}$ avaliação).

O intervalo entre as duas avaliações variou de 5 meses até 7 meses e 23 dias.

O número de crianças do sexo masculino foi de 18 e do feminino 15 .

As idades gestacionais das 33 crianças foram adequadas no caso de 31, isto é, era de 37 até 42 semanas (inclusive). Duas crianças tiveram idade ao nascimento menor, de 36 semanas e 36 semanas e 4 dias, respectivamente, e neste caso foi realizado o ajuste recomendado pelos autores do TTDD-II (ARCHER et al., 1992).
Tivemos duas duplas de gêmeos que nasceram no período considerado adequado, isto é, entre 37 e 42 semanas.

O peso de nascimento das crianças variou entre $1970 \mathrm{~g}$ a $4280 \mathrm{~g}$.

Quanto à situação sócio-econômica. o grupo estudado demonstrou ser homogêneo: não houve família alguma que tivesse pontuação menor do que 45 (ISSLER \& GIUGLIANI, 1997). Deste modo, todas se enquadram, no mínimo, na categoria baixa superior. Assim, é possível considerar que todas as crianças que participaram do estudo partiram de um mesmo patamar mínimo. A pontuação obtida pelas 31 famílias (havia dois pares de gêmeos de cada criança) variou de 45 a 52. Houve uma família que alcançou 45 pontos e todas as outras (em número de 30) obtiveram de 46 a 52.

As classificações obtidas pelas crianças referentes às áreas de linguagem (adequado, cautela e atraso) foram as seguintes (Tab. 1):

Na primeira avaliação 26 crianças tiveram desempenho adequado (78,78\%), e 7 (21,22\%) não adequado. É importante lembrar que cautela não pode ser considerado verdadeiramente um risco, tal como atraso. Assim, estaríamos fazendo a análise mais pessimista, o que no entanto, é mais prudente, pois faz com que o profissional de saúde e o educador fiquem mais atentos à situação da criança.

Na segunda avaliação da mesma área, linguagem, obteve-se 28 adequações $(84,84 \%)$, e $5(15,15 \%)$ cautelas. Não houve atrasos, nem 
cautela + atraso. Percebe-se, portanto, que houve melhora de um período em relação ao outro.

Quanto à área pessoal-social as crianças se comportaram da seguinte maneira (Tab. 2):

as creches também não diferem nesta área (Tab. 4).

Assim, os dados referentes às avaliações de linguagem das 33 crianças foram agrupados (Quadro 1) e trabalhados conjuntamente, fazendo-se a comparação da primeira com a segunda avaliação através do teste dos sinais (SIEGEL, 1975). Para a realização do teste dos sinais, nas duas áreas, cada criança foi comparada consigo mesma nos dois momentos em que foi avaliada. Considerou-se empate quan-

Na primeira avaliação 26 (78,78\%) crianças estavam adequadas e 7 (21,22\%) apresentavam cautela ou atraso. Na segunda o resultado foi excelente: todas 33 (100\%) estavam adequadas.

Com a finalidade de verificar se havia diferença entre as três creches, a área de linguagem foi analisada usando-se as categorias adequada e cautela somada à de atraso usandose o teste exato de Fisher (SIEGEL, 1975). O resultado não foi significativo e a partir deste momento os dados referentes às 3 creches foram trabalhados juntos (Tab. 3).

O mesmo procedimento foi repetido quanto à área pessoal-social obtendo-se também resultados não significativos, ou seja,

Tabela 3. Interpretação dos Itens de Linguagem da $1^{\text {a }}$ Avaliação de Crianças de 3 Creches. São Paulo, 2001.

\begin{tabular}{cccc}
\hline Creche & Adequada & Cautela + Atraso & Total \\
\hline A & 14 & 3 & 17 \\
B & 7 & 2 & 9 \\
C & 5 & 2 & 7 \\
\hline Total & $26(78,8 \%)$ & $7(21,2 \%)$ & $33(100 \%)$ \\
\hline Teste exato de Fischer & & \\
Risco alfa: $5 \%$. \\
Creche B X C: $\mathrm{P}=0,607$. \\
Creche A X BC: $\mathrm{P}=0,463$.
\end{tabular}

do esta se manteve adequada nas duas avaliações, sinal positivo quando ela teve uma interpretação diferente de adequada na $1^{\mathrm{a}}$ avaliação e mudou para adequada na $2^{\mathrm{a}}$, e sinal negativo quando ocorreu o inverso.

$\mathrm{Na}$ área de linguagem houve 21 empates, 7 sinais positivos e 5 sinais negativos, não significativo ( $\mathrm{p}=0,387)$. Este resultado demonstra que as habilidades de linguagem das crianças não se modificaram entre os dois períodos avaliados: o bom desempenho medido em primeiro momento repetiu-se no segundo. Pode-se afirmar que as crianças freqüentarem creches não prejudicou seu desenvolvimento, e isto numa área em que são muito dependentes do comportamento dos adultos (BURCHINAL et al., 1996).

Tabela 4. Interpretação dos Itens da Área Pessoal-Social da $1^{\text {a }}$ Avaliação de Crianças de 3 Creches. São Paulo, 2001.

\begin{tabular}{cccc}
\hline Creche & Adequada & Cautela + Atraso & Total \\
\hline A & 12 & 5 & 17 \\
B & 8 & 1 & 9 \\
C & 6 & 1 & 7 \\
\hline Total & $26(78,8 \%)$ & $7(21,2 \%)$ & $33(100 \%)$ \\
\hline Teste exato de Fischer & & \\
Risco alfa: $5 \%$. \\
Creche B X C: $\mathrm{P}=0,700$. \\
Creche A X BC: $\mathrm{P}=0,224$.
\end{tabular}


Quadro 1. Resultados Emparelhados das Avaliações da Área de Linguagem pelo TTDD-II de Crianças em 3 Creches. São Paulo, 2001.

\begin{tabular}{|c|c|c|c|}
\hline Crianças & $1^{\mathrm{a}}$ Avaliação & $2^{\mathrm{a}}$ Avaliação & Classificação \\
\hline 1. & Adequada & Adequada & Empate \\
\hline 2. & 1 Cautela & Adequada & + \\
\hline 3. & 1 Cautela & Adequada & + \\
\hline 4. & 1 Cautela +1 Atraso & Adequada & + \\
\hline 5. & Adequada & Adequada & Empate \\
\hline 6. & Adequada & Adequada & Empate \\
\hline 7. & Adequada & Adequada & Empate \\
\hline 8. & Adequada & Adequada & Empate \\
\hline 9. & Adequada & Adequada & Empate \\
\hline 10. & Adequada & Adequada & Empate \\
\hline 11. & Adequada & Adequada & Empate \\
\hline 12. & Adequada & Adequada & Empate \\
\hline 13. & Adequada & Adequada & Empate \\
\hline 14. & Adequada & Adequada & Empate \\
\hline 15. & Adequada & Adequada & Empate \\
\hline 16. & Adequada & Adequada & Empate \\
\hline 17. & Adequada & Adequada & Empate \\
\hline 18. & 1 Cautela & Adequada & + \\
\hline 19. & Adequada & Adequada & Empate \\
\hline 20. & Adequada & 1 Cautela & - \\
\hline 21. & Adequada & Adequada & Empate \\
\hline 22. & 1 Cautela +1 Atraso & Adequada & + \\
\hline 23. & Adequada & Adequada & Empate \\
\hline 24. & Adequada & 1 Cautela & - \\
\hline 25. & Adequada & 1 Cautela & - \\
\hline 26. & Adequada & Adequada & Empate \\
\hline 27. & Adequada & Adequada & Empate \\
\hline 28. & 1 Cautela & Adequada & + \\
\hline 29. & Adequada & 1 Cautela & - \\
\hline 30. & Adequada & Adequada & Empate \\
\hline 31. & Adequada & Adequada & Empate \\
\hline 32. & Adequada & 1 Cautela & - \\
\hline 33. & 1 Cautela & Adequada & + \\
\hline
\end{tabular}

Teste dos sinais; $\mathrm{P}=0,387$.

Na área pessoal-social houve 26 empates, 7 sinais positivos e nenhum negativo. $\mathrm{O}$ resultado dos testes foi significante $(\mathrm{p}=0,008)$ (Quadro 2).
Como esta também é uma área em que as crianças dependem da atuação dos adultos, consideramos o resultado muito importante: as crianças freqüentarem a creche não prejudi- 
Quadro 2. Resultados Emparelhados das Avaliações da Área Pessoal-Social pelo TTDD-II de Crianças em 3 Creches. São Paulo, 2001.

\begin{tabular}{|c|c|c|c|}
\hline Criança & $1^{a}$ Avaliação & $2^{\mathrm{a}}$ Avaliação & Classificação \\
\hline 1. & Adequada & Adequada & Empate \\
\hline 2. & 1 Cautela & Adequada & + \\
\hline 3. & 1 Cautela & Adequada & + \\
\hline 4. & Adequada & Adequada & Empate \\
\hline 5. & 1 Cautela & Adequada & + \\
\hline 6. & 1 Cautela & Adequada & + \\
\hline 7. & Adequada & Adequada & Empate \\
\hline 8. & 1 Atraso & Adequada & + \\
\hline 9. & Adequada & Adequada & Empate \\
\hline 10. & Adequada & Adequada & Empate \\
\hline 11. & Adequada & Adequada & Empate \\
\hline 12. & Adequada & Adequada & Empate \\
\hline 13. & Adequada & Adequada & Empate \\
\hline 14. & Adequada & Adequada & Empate \\
\hline 15. & Adequada & Adequada & Empate \\
\hline 16. & Adequada & Adequada & Empate \\
\hline 17. & Adequada & Adequada & Empate \\
\hline 18. & Adequada & Adequada & Empate \\
\hline 19. & Adequada & Adequada & Empate \\
\hline 20. & 1 Cautela & Adequada & + \\
\hline 21. & Adequada & Adequada & Empate \\
\hline 22. & Adequada & Adequada & Empate \\
\hline 23. & Adequada & Adequada & Empate \\
\hline 24. & Adequada & Adequada & Empate \\
\hline 25. & Adequada & Adequada & Empate \\
\hline 26. & Adequada & Adequada & Empate \\
\hline 27. & Adequada & Adequada & Empate \\
\hline 28. & Adequada & Adequada & Empate \\
\hline 29. & 1 Cautela & Adequada & + \\
\hline 30. & Adequada & Adequada & Empate \\
\hline 31. & Adequada & Adequada & Empate \\
\hline 32. & Adequada & Adequada & Empate \\
\hline 33. & Adequada & Adequada & Empate \\
\hline
\end{tabular}

Teste dos sinais; $\mathrm{P}=0,008$ *.

cou seu desenvolvimento, talvez até o tenha $o$ melhorado devido às possibilidades de interação entre crianças e adultos e crianças e seus pares. Sem dúvida, não pode ser negligenciada a influência da família, da qual o bom desempenho na primeira avaliação é uma evidência. Mas a continuidade desta estimulação deve ser buscada na instituição. Assim, a expli- 
cação deste resultado deve se voltar para os recursos humanos de que dispunham. É o que faremos a seguir.

Ao analisarmos a estrutura (BURCHINAL et al., 1996; BURCHINAL et al., 2000) das creches, ou seja, a proporção adulto/criança nos grupos que participaram da pesquisa, e compararmos com o preconizado pela Secretaria do Menor (SÃO PAULO, 1992) obtivemos a tabela abaixo (Tab. 5).

Observa-se que, nas creches estudadas, a estrutura está adequada, sendo em alguns grupos a proporção adulto/criança até menor do que o recomendado, o que reafirma o bom padrão de cuidado/educação dessas creches. Além disto, alguns grupos possuem funcionárias volantes que não foram incluídas na tabela mas que também auxiliam no cuidado/educação.
Mais ainda: as crianças são cuidadas sempre pelas mesmas educadoras, que ficam responsáveis por grupos pequenos, o que é importante para seu bem-estar emocional e, consequentemente, para a sua disponibilidade para se desenvolverem (BOWLBY, 1984; HONIG, 2001).

No que diz respeito à situação de processo, temos somente um indicador até o momento e que se refere à creche B. Pesquisou-se de que modo as educadoras percebiam o conversar com crianças de 0 a 3 anos de idade (SHIBAYAMA, 2001). Percebeu-se que, para as educadoras, conversar é uma atividade executada e valorizada. As educadoras constróem intencionalmente o conversar e este ato é, inclusive, discutido em suas reuniões de trabalho que são periódicas.

Tabela 5. Proporção Educador/Criança em 3 Creches. São Paulo, 2001.

\begin{tabular}{ccccc}
\hline Faixa etária & $\begin{array}{c}\text { Proporção } \\
\text { recomendada }\end{array}$ & $\begin{array}{c}\text { Proporção real } \\
\text { Creche A }\end{array}$ & $\begin{array}{c}\text { Proporção real } \\
\text { Creche B }\end{array}$ & $\begin{array}{c}\text { Proporção real } \\
\text { Creche C }\end{array}$ \\
\hline 0 a 1 ano e 11 meses & $1 / 5$ & $1 / 5$ & $1 / 4$ & $1 / 5$ \\
2 anos a 2 anos e 11 meses & $1 / 10$ & $1 / 4$ & $1 / 6$ & Não se aplica \\
\hline
\end{tabular}

\section{CONSIDERAÇÕES FINAIS}

Dividiremos o item de considerações finais em duas sub-áreas: uma, relativa aos resultados obtidos das avaliações das crianças propriamente ditas; outra referente aos cuidados metodológicos que foram adotados e que são tão importantes quanto o resultado propriamente dito.

No que diz respeito à primeira, foi possível relacionar os bons desempenhos obtidos pelas crianças às condições estruturais, e talvez às de processo, apresentadas pelas creches. Sem dúvida, a influência da família também se deu. Deste modo, foi possível perceber que uma "boa creche", ou seja, uma que apresenta padrões de qualidade objetivos e mensuráveis como, por exemplo, a proporção de crianças por educador, dá continuidade à estimulação começada pela família. É possível até que a creche propicie uma situação de desenvolvimento verbal à criança mais adequada do a família. No entanto, definir a influência de cada uma dessas duas esferas, família e creche, exige outros estudos.

É imprescindível levar em consideração, ao se lidar com a questão do cuidado na creche, a quantidade e a qualificação do pessoal adulto disponível. Esta questão precisa permanecer sempre em foco.

No que diz respeito a estudos realizados na área, não é mais admissível que a instituição creche seja simplesmente nomeada, sem que se apresente ao leitor sua conformação e seu modo de agir, ainda que brevemente, dado que, sob o nome "creche”, há modos muito distintos de educar e cuidar. 
Em relação aos cuidados metodológicos, tivemos a possibilidade e o intento de rastrear todas as situações julgadas deletérias à avaliação da criança. Assim, eventuais cautelas e atrasos seriam, na medida do possível, devidos a própria criança, e não a quaisquer outros fatores que mascaram o desenvolvimento infantil pleno.

Ressaltamos que o TTDD-II foi criado para de ser aplicado em consultórios e ambulatórios, enfim, em locais nos quais se conta com a presença dos familiares da criança. A presença da mãe, ou de outro adulto significativo, modifica o comportamento da criança (BOWLBY, 1984). Assim, ao realizarmos a avaliação em ambiente do qual não se dispunha da presença da mãe, ou de algum outro adulto significativo para a criança, foi necessário tomar estes cuidados para garantir a serenidade e sentimento de confiança da criança.

Deste modo, o tempo necessário para aplicação aumentou muito. A afirmação de que o tempo necessário para aplicação é de 15 a 20 minutos (O’HARA et al., 1998) não se aplica à avaliação dessas crianças. Outra situação importante foi o auxílio das educadoras infantis para que a avaliação se desse. Foi mais do que "criar condições” para ajudar na obtenção das informações. Nas três creches, as educadoras são fixas, isto é, permanecem sempre com as mesmas crianças. Isto é feito com o propósito de favorecer a ligação afetiva entre criança e educador, condição útil para o favorecimento do desenvolvimento das crianças. Assim, as educadoras tornam-se o que BOWLBY (1984) nomeia “cuidadores secundários" e exercem, em parte, o papel que caberia à mãe (ou ao cuidador pri- mário) durante a aplicação do TTDD-II. As educadoras executam um papel importante servindo como base logística para os pesquisadores devido à sua posição dentro da instituição e a sua posição como base afetiva para as crianças.

Finalmente, salientamos que seria indicado reproduzir este estudo em condições diferentes. Por exemplo: se as crianças avaliadas proviessem de lares nos quais não dispõem de um ambiente propício ao desenvolvimento, e passassem a freqüentar creches nas quais têm esta oportunidade. Outros desenhos também poderiam ser feitos. De qualquer modo, sabese que as condições ambientais são fundamentais para o desenvolvimento infantil. É necessário que tais parâmetros, sejam incorporados a todas as creches.

\section{AGRADECIMENTOS}

Agradecemos a inúmeras pessoas e instituições, dentre elas, Prof ${ }^{a}$. Dra. Márcia R. M. Pedromônico da Disciplina de Distúrbios da Comunicação Humana da Universidade Federal de São Paulo - Escola Paulista de Medicina pela permissão do uso do formulário do TTDDII, traduzido por ela e sua equipe, bem como por sua disponibilidade de discussão, à Coordenadoria de Assistência Social (COSEAS) da USP, aos responsáveis pelas crianças, às próprias crianças e à Fernanda Guedes de Lima, acadêmica de enfermagem, que durante o período da pesquisa participou voluntariamente de todas as atividades, incluindo coleta e análise dos dados, elaboração dos relatórios e participação dos congressos.

Abstract: Supervision and promotion of infantile development are important health actions and must
be performed in day care centers. Thus, the child can develop, mainly in areas where he/she depends
on the adult's support, such as in the language and personal-social ones. The objective of this study
was to evaluate language and personal-social abilities in children enrolled in three high-quality day
care centers in the city of São Paulo. Children from 0 to 36 months were evaluated by the
Denver II Selection Test of Development (TTDD II) in 2 different moments, with a 5-month interval.
They all had an adequate socioeconomic level. Ethic cares according to the Brazilian 
legislation were considered. Thirty-three children were evaluated: 19 from the day care center A, 9 from B and 3 from C. Ages in the first evaluation ranged from 4 months and 23 days to 2 years, 7 months and 11 days. In the second evaluation, ages ranged from 10 months and 20 days to 2 years, 10 months and 8 days. Eighteen were males and fifteen females. Two children had their gestational age corrected. Two couples were twins who were born in an adequate gestational period (more than 37 weeks). Weight at birth varied from 1,970 to 4,280 g. Regarding language and personalsocial areas, the obtained classifications in the first evaluation were analyzed by the Fisher's test (risk $\mathrm{a}=0.05$ ) with a non-significant result. Thus, the population of the three day care centers was unified. In the second evaluation, children's abilities improved in the two areas. The results of each child were paired and analyzed by the Sign's test (risk a $=0.05$ ): non-significant results were obtained in the language area but they were significant in the personal-social area $(\mathrm{p}=0.008)$. The day care centers must have contributed to the obtention of these results. Structural factors (proportion adult/ child, small groups of children) of the three day care centers were adequate.

Key words: child day care centers; child rearing; child care; pediatric nursing; child development.

\section{REFERÊNCIAS BIBLIOGRÁFICAS}

ALVAREZ, M.L.; WURGAFT, F.; SALAZAR M.E. Mediciones del nível socioeconomico bajo urbano en famílias con lactante desnutrido. Archivos Latinoamericanos de Nutricion, 32:650-652, 1982.

AUSTRÁLIA. CONSELHO NACIONAL DE CREDENCIAMENTO DE CRECHES. Priorizando as crianças: sistema de promoção de qualidade credenciamento. s/d, (mimeo), 1993.

ARCHER, P.; FRANKENBURG, W.K.; DODDS, J.; BRESNICK, B.; MASCHKA, P.; EDELMAN, N.; SHAPIRO, H. Denver II Training Manual. Denver, 1992.

BARNARD, K.E.; ERIKSON, M.L. Como educar crianças com problemas de desenvolvimento. Porto Alegre: Globo, 1978. (Cap. 6: Avaliação do desenvolvimento. e funcionamento da criança).

BOWLBY, J. Apego. São Paulo: Martins Fontes, 1984.

BRÊTAS, J.R.d.S. Avaliação psicomotora de crianças de 5 a 7 anos de idade, que freqüentam a creche “Maria Aparecida Carlini”, Jardim Sabiá , Município de São Paulo, São Paulo. São Paulo, 1991. 123 p. [Dissertação de Mestrado Escola Paulista de Medicina].

BRÊTAS, J.R.d.S.; SILVA, M.d.G. A aplicação do teste de triagem de desenvolvimento de Denver pelo enfermeiro pediatra: relato de caso. Acta Paul. Enf., 8(4): 9-16, 1995.

BURCHINAL, M.R.; ROBERTS, J.E.; NABORS, L.A.; BRYANT, D.M. Quality of center child care and infant cognitive and language development. Child Development, 67(2): 606620, 1996.
BURCHINAL, M.R.; ROBERTS, J.E.; RIGGINS J.R., R.; ZEISEL, S.A.; NEEBE, E.; BRYANT, D. Relating quality of center-based child care to early cognitive and language development longitudinally. Child Development, 71(2): 339357, 2000.

CARVALHO, S.; SCARPA, R. Encruzilhada de vozes: como crianças de 2 a 5 anos se comunicam e como os adultos podem ajudar. Avisalá, 1(4):8-13, 2000.

CUNHA, H.L. Desenvolvimento de crianças atendidas no hospital de pediatria da Universidade Federal do Rio Grande do Norte no primeiro ano de vida: aplicação do teste de Denver II em ambulatório. São Paulo, 2000. 82p. [Dissertação de Mestrado - Escola Paulista de Medicina/Universidade Federal de São Paulo].

DURMAZLAR, N.; OZTURK, Ç.; URAL, B.; KARAAGAOGLU, E.; ANLAR, B. Turkish children's performance on Denver II: effect of sex and mother's education. Develop. Med. and Child Neurology, (40): 411-416, 1998.

FERREIRA, A .M.d.A. Avaliação nutricional e do desenvolvimento de crianças de 0 a 3 anos de idade que freqüentam creches conveniadas com a Secretaria Municipal do Bem-Estar Social de São Paulo na regional da Lapa. São Paulo, 1989. 150p. [Dissertação de Mestrado - Escola Paulista de Medicina].

FISBERG, M.; PEDREMÔNICO, M.R.; BRAGA, J.A. P.; FERREIRA, C.; PINI, S.C.C.; CAMPOS, S.O.; LEMES, S.; SILVA, R.S.; ANDRADE, T.M. Comparação do desempenho de pré-escolares, mediante teste de desenvolvimento de Denver, antes e após intervenção nutricional. Rev. Ass. Med. Brasil. 43(2): 99-104, 1997. 
HONIG, A.S. Secure relatioships: nurturing infant/ toddler attachment in early care settings. Washington, National Association for the education of young children, 2001. 78p.

HUGUES, M.d.S. Avaliação, através do exame físico e teste de Gesell, de aspectos de saúde de crianças de 2 a 44 meses de idade, que freqüentam a creche Maria Aparecida Carlini, Jardim Sabiá, Bairro do município de São Paulo, São Paulo. São Paulo, 1986. 95p. [Dissertação de Mestrado - Escola Paulista de Medicina].

ISSLER, R.M.S.; GIUGLIANI, E.R.J. Identificação de grupos vulneráveis à desnutrição infantil pela medição do nível de pobreza. Jornal de Pediatria, 73:101-105, 1997.

KAKEHASHI, S. Avaliação de alguns aspectos do desenvolvimento motor grosso e fino, adaptativo, pessoal social e de linguagem das crianças de 2 a 6 anos de idade da creche "Maria Aparecida Carlini”, Jardim Sabiá, São Paulo, SP, 1987. São Paulo, 1990. 194p. [Dissertação de Mestrado - Escola Paulista de Medicina].

MINISTÉRIO DA SAÚDE. Atendimento integrado à saúde e desenvolvimento da criança: módulo 1. Cartão da criança. Brasília, 1995. 38p.

MINISTÉRIO DA SAÚDE. Saúde da criança: acompanhamento do crescimento e desenvolvimento infantil. Brasília, 2002. 100p. (Série Cadernos de Atenção Básica, no. 11).

O'HARA, M.T.; CHURCH, C.C.; BLATT, S.D. Homebased developmental screening of children in foster care. Pediatric Nursing, 24(2):113-117, 1998.

ORGANIZACIÓN PANAMERICANA DE LA SALUD. Promoción del crescimiento y desarollo integral de niños y adolescentes: módulos de aprendizaje. Washington, 1999. 142p.
PEDREMÔNICO, M.R.M.; BRAGATTO, E.L.; STROBILIUS, R. Denver II. São Paulo, 1999. [formulário].

SÃO PAULO (Estado). Secretaria do Menor. Creche/Pré-escola/Secretaria do Menor. São Paulo, 1992. 144p. (Série Secretaria do Menor: 3 anos de experiência).

SHIBAYAMA, R.V.d.S. O conversar com crianças de creches segundo suas educadoras/ cuidadoras. São Paulo, 2001. 68p. [Dissertação de Mestrado - Escola de Enfermagem da Universidade de São Paulo].

SIEGEL, S. Estatística não-paramétrica para as ciências do comportamento. São Paulo: Mc Graw- Hill, 1975.

TOLEDO, M.N.C.d. Mãe! E agora, o que é que falo? Avisalá, 1(2):27-30, 2000.

TURNER, S.B. Caretaking of children's souls? Teaching the deep song. Young Children, 55(1): 31- 3, 2000.

VERÍSSIMO, M.D.LaÓR; SIGAUD, C.H.d.S. O ser criança. In: VERÍSSIMO, M.D.LaÓR; SIGAUD,

C.H.d.S. (Orgs.). Enfermagem pediátrica: o cuidado de enfermagem à criança e ao adolescente. São Paulo: EPU, 1996. p.11-14.

WHALEY, L.F.; WONG, D.L. Enfermagem pediátrica: elementos essenciais à intervenção efetiva. $2^{\text {a }}$ ed. Rio de Janeiro: Guanabara / Koogan, 1989.

WONG, D.L. Whaley \& Wong: Enfermagem pediátrica, elementos essenciais à intervenção efetiva. $5^{\mathrm{a}}$ ed. Rio de Janeiro: Guanabara / Koogan, 1999.

Recebido em 24/02/2003

Modificado em 20/03/2003

Aprovado em 25/03/2003 